\title{
Multilayer perceptron for gait type classification based on inertial sensors data
}

\author{
Damian Szczepański \\ Rzeszów University of Technology al. Powstancow Warszawy 12, 35-959 Rzeszów, Poland \\ Email: dszczepanski@prz.edu.pl
}

\begin{abstract}
In this study, the gait type classification process is considered. Input data are obtained by using the inertial sensors tracking system. Three types of gait are recorded: normal walk, tiptoeing and walk retaining long stance phase. Two data set types, describing the registered motion, are prepared. The most significant input features are selected by means of the sensitivity analysis (SA) procedure. The classification process is conducted using multilayer perceptron (MLP) with various structures. The classification accuracy of the network is computed with the use of a cross validation procedure. The obtained results show that the successful classification of presented gait types can be achieved using relatively simple MLP architecture.
\end{abstract}

\section{INTRODUCTION}

$\mathbf{N}$ OWADAYS, as the society is getting older, the illnesses typical for the elderly age, are becoming more and more meaningful part of modern medicine. In turn, the doctors need immediate and accurate methods in everyday diagnosing. The analysis of human gait from the very beginning has been a basic method in rehabilitation and early locomotive disease detection. Nowadays, Parkinson's disease (PD) is most common and onerous dysfunction of medicine [1]. At the early stage, PD does not show any significant symptoms, except for small gait and posture changes. Typical gait modification relies on the increasing foot contact in stance phase (shuffling steps), forward-flexed posture and limbs tremor. In order to treat PD effectively, the rehabilitation and exercises should be employed at the very early stage of disease detection. The technology progress in human body motion capture provides new solutions which could be used in human gait and posture analyses.

Since the beginning, the optical systems have been the basic tool in human motion capture. However, due to its complicity, their usage can be very complicated on a daily basis. One of the most promising approaches is the inertial sensors technology, which is simpler in usage and comparable in accuracy with the optical systems. Inertial systems have been used in many fields of medical diagnose assistance[2], i.e.: cerebral palsy [3], [4], determining gait defects, sport medicine [5]. Flexibility advantage of such system is very useful in rehabilitation [6], exercise motivation [7] and automatic exercise advising [8].

The main purpose of this article is to explore the classification problem of gait which is registered on the basis of the motion parameters such as joint angles, segments velocity and accelerations values. The motion acquisition is conducted by using Xsens MVN Biomech suit (MVN) [9] which is based on inertial measure units (IMU) wirelessly connected to the computer. In the classification process, the MLP classifier is used and the best architecture with highest accuracy is chosen. In current study, one also considers the problem of input features' selection. Therefore, the SA procedure is utilized to determine the most significant gait attributes. MLP classification performance is assessed with reduced number of inputs.

All simulations, the classification process and the IMU synthesis are conducted by using Matlab [10], DTReg [11] and MVN Studio [12] software.

The paper is composed of the following sections. Section II presents the input data used in this work. In Section III, the problem statement is formulated and the approaches utilized in the classification process are shortly described. Sections IV and V outline the experiments' settings and the obtained results, respectively. Section VI concludes the paper.

\section{INPUT DATA}

\section{A. Data acquisition}

The registration process is conducted by using MVN which utilizes 17 inertial sensors attached to each body segment (see Fig. 1).

Two subjects (M: 90kg, $180 \mathrm{~cm}, 29$ years, M: $75 \mathrm{~kg} 181$ $\mathrm{cm}, 26$ years) take part in the experiment. They are asked to walk in three different gait manners: (a) - normal walk with natural speed, (b) - tiptoeing and (c) - walk retaining long stance phase and very small distance between foot and ground in swing phase. The (c) type of gait is similar to PD walk. Registration is repeated 20 times per gait type by each person, which gives 120 data series for all gait types.

One gait cycle, which is one step, is defined as a time between heel ground contacts. Fig. 2 presents a joint angle registered within a single gait cycle.

\section{B. Data type}

Raw data (accelerometer, gyroscope, magnetometer values), which are acquired directly from IMU sensors during registration process, are synthesized by MVN Studio Software. All joint angles, segment accelerations, segment velocity, angular segment accelerations and angular segment velocity are calculated. Each parameter has three values in each dimension what, in turn, gives 612 values per one time frame data. Sample screen-shot from MVN Studio with rendered subject avatar and plotted joint angles are presented in Fig. 3. 


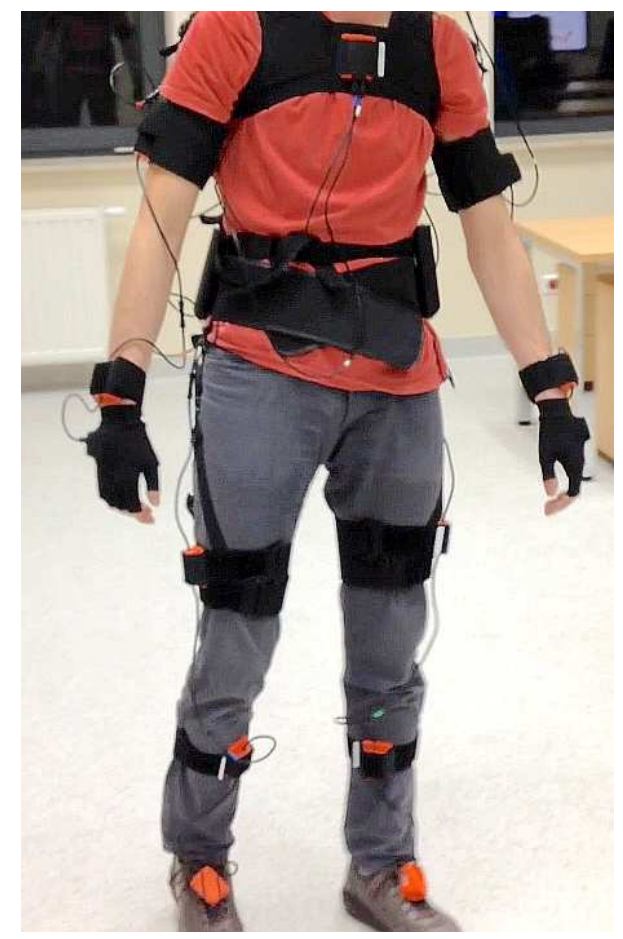

Fig. 1. Inertial sensors attached to body segments.

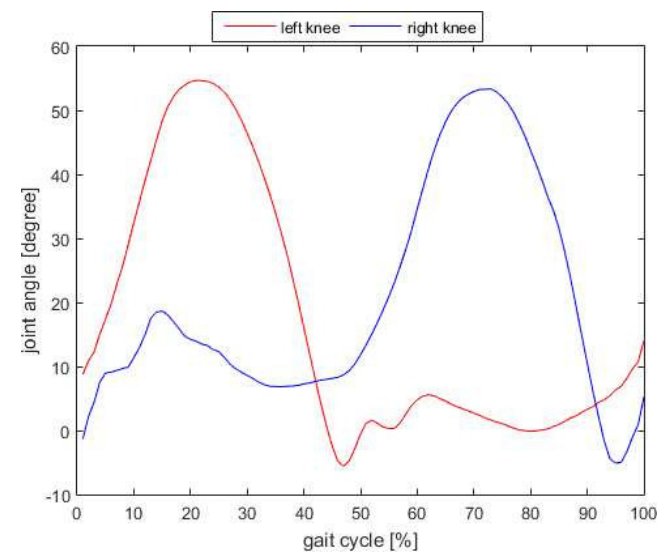

Fig. 2. Flexion - extension knee angle in one gait cycle: left knee (red), right knee (blue).

\section{Input vectors}

For the purpose of the comparison, two data sets are prepared from the gait recordings. The first data set is represented by statistical values (SV): maximum value, minimum value, mean and standard deviation, which are calculated from acceleration values of 17 body segments. Every IMU accelerometer measure signal in $x, y, z$ axis what in result gives 204 values (4 statistical values of 17 segments in 3 axis). These are: pelvis, thoracic spine, head, right shoulder, right upper arm, right forearm, right hand, left shoulder, left upper arm, left forearm, left hand, right upper leg, right lower leg, right foot, left upper leg, left lower leg, and left foot. An exemplary single body segment, i.e. left upper leg, is illustrated in Fig. 3 (red curve).

The second data set is represented by the normalcy index (NI) parameters commonly used in gait analyses [13]. The 15 values proposed in [14] are determined: time of toe off, walking speed, mean pelvic tilt, range of pelvic tilt, mean pelvic rotation, minimum hip flexion, range of hip flexion, peak abduction in swing, mean hip rotation in stance, knee flexion at initial contact, time of peak knee flexion, range of knee flexion, peak dorsiflexion in stance phase, peak dorsiflexion in swing, and mean foot progression angle. All calculations are conducted in Matlab software.

Three types of gait described in subsection II-A impose three class classification problem.

\section{Application OF MLP NETWORK TO GAit CLASSIFICATION PROBLEM}

\section{A. Problem statement}

For the input data set described in Section II, the task is to find the optimal MLP architecture with respect to the highest prediction accuracy (Acc) of the model. The accuracy is calculated as a sum of true positives and true negatives divided by number of all input vectors. The experiment is repeated 10 times and after all tests, the average value and the standard deviation (Std) of Acc are calculated. The accuracy in each test trial is computed using a 10 -fold cross validation procedure. Furthermore, an optimal subset of input features is found with the use of the SA procedure. As in the case of all input attributes, the highest Acc of MLP is determined for reduced data set.

\section{B. Multilayer perceptron}

MLP is the feedforward neural network [15], [16], i.e. the model where the input signal is fed forward through a number of layers [17]. There are three types of layers in MLP: an input layer, one or more hidden layers and an output layer. The input layer consists of the nodes which are the features of an input vector. Each of $i=1, \ldots, n$ input features is connected to a hidden layer neuron activated as follows

$$
y_{j}^{(1)}=f\left(\sum_{i=1}^{n} w_{j i}^{(1)} x_{i}+b_{j}^{(1)}\right),
$$

where $f(\cdot)$ is the activation function, $\mathbf{w}_{j}^{(1)}=\left[w_{j 1}^{(1)}, \ldots, w_{j n}^{(1)}\right]$ is the weight vector of the $j$ h hidden neuron, $\mathbf{x}=\left[x_{1}, \ldots, x_{n}\right]$ is an input vector and $b_{j}^{(1)}$ denotes the bias of the $j$ h hidden neuron. The outputs $y_{j}^{(1)}$ of the hidden neurons are linearly combined with the second layer weights $w_{k j}^{(2)}$ and the bias $b_{k}^{(2)}$ and, after activation, create an output of the $k$ th neuron in the second layer

$$
y_{k}^{(2)}=f\left(\sum_{j=1}^{m} w_{k j}^{(2)} y_{j}^{(1)}+b_{k}^{(2)}\right) .
$$




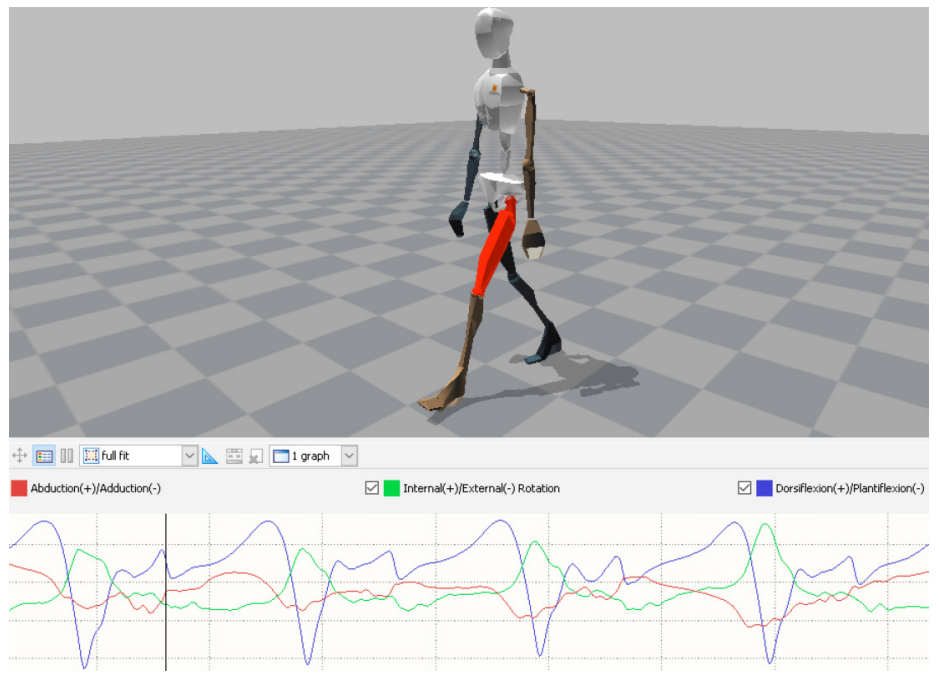

Fig. 3. MVN Studio screen-shot presenting rendered avatar with selected upper leg segment and plotted knee angles.

In (1) and (2), $f(\cdot)$ should be selected in the way it is continuous, differentiable and monotonically increasing. $f(a)=$ $\frac{1}{1+e^{-\lambda a}}$ is the most commonly used activation function. The number of hidden layers and the number of neurons in each hidden layer must be optimized in order to maximize MLP's prediction accuracy. In this study, scaled conjugate gradient algorithm is used for MLP training.

\section{Sensitivity analysis procedure}

SA procedure steps separately through a single feature in each iteration. It randomly permutes its values across the rows of the data set. The values of this feature remain the same but they are randomly moved to different rows. Then the accuracy the network is evaluated on the modified data set. If the feature is important, randomizing the order of its values greatly degrades the accuracy of the predictions. If the feature is not valuable in prediction, the order of its values has little or no influence on the accuracy of the network. Last step is to determine the rank of the importance of features based on the amount of degradation that occurred by randomizing their values and to scale the scores so the most important feature has a relative importance of 100 .

\section{EXPERIMENTS}

MLP used in experiment has 3 outputs (one for each gait type). The number of neurons in the hidden layer is taken from the set $\{2, \ldots, 10\}$. The linear, logistic and tangent activation functions are utilized for MLP training. In the first part of the experiment, MLP is trained by using all input variables both for SV and NI data. As a result, the best network architectures for both data sets are chosen. The second part of the experiments relies on applying the SA procedure to select features for input neurons' representation. Table I and II present the importance of features for NI and SV data set, respectively. Due to the fact that $\mathrm{SV}$ data vector is composed of 204 attributes, only 7 significant features are presented. For the
TABLE I

FIRST 7 MOST IMPORTANT NI DATA SET FEATURES.

\begin{tabular}{|l|c|}
\hline \multicolumn{1}{|c|}{ Parameter } & Importance \\
\hline peak body dorsiflexion in stance phase & 100.00 \\
\hline knee range flexion & 61.90 \\
\hline peak body dorsiflexion in swing phase & 48.87 \\
\hline range of hip flexion & 9.61 \\
\hline mean foot progression angle & 7,28 \\
\hline walking speed & 5,45 \\
\hline mean hip rotation in stance & 3,21 \\
\hline
\end{tabular}

TABLE II

FIRST 7 MOST IMPORTANT SV DATA SET FEATURES.

\begin{tabular}{|l|c|}
\hline \multicolumn{1}{|c|}{ Parameter } & Importance \\
\hline mean right foot acceleration in $\mathrm{Z}$ axis & 100.00 \\
\hline mean acceleration of right thigh in $\mathrm{Y}$ axis & 69,79 \\
\hline mean left foot acceleration in $\mathrm{Z}$ axis & 69,75 \\
\hline mean right foot acceleration in X axis & 48,72 \\
\hline mean left foot acceleration in $\mathrm{Y}$ axis & 38,11 \\
\hline mean left foot acceleration in X axis & 35,08 \\
\hline standard deviation of pelvis tilt acceleration in $\mathrm{Z}$ axis & 18,75 \\
\hline
\end{tabular}

second part of the experiment, only 3 most significant features of SV and NI dataset are selected. The classification process is repeated using reduced MLP and best network architectures are chosen.

\section{Results}

\section{A. Result for SV dataset}

For MLP with 204 inputs, the highest accuracy (99.18\%) is achieved for the network with 2 hidden neurons. Using only mean right foot acceleration in $\mathrm{Z}$ axis (importance 100), mean acceleration of right thigh in $\mathrm{Y}$ axis (importance 69.79), mean acceleration of left foot in $\mathrm{Z}$ axis (importance 69.74), 
TABLE III

RESULTS OF SV DATASET CLASSIFICATION.

\begin{tabular}{|l|c|c|c|}
\hline \multicolumn{1}{|c|}{ Used parameter } & Neurons & Acc & Std \\
\hline all parameters & 2 & $99.18 \%$ & $0.11 \%$ \\
\hline $\begin{array}{l}\text { mean right foot acceler. in Z axis, } \\
\text { mean acceler. of right thigh in Y axis, } \\
\text { mean left foot acceler. in Z axis }\end{array}$ & 2 & $100.00 \%$ & $0.00 \%$ \\
\hline mean right foot acceler. in Z axis & 2 & $100.00 \%$ & $0.00 \%$ \\
\hline mean acceler. of right thigh in Y axis & 3 & $91.14 \%$ & $0.93 \%$ \\
\hline mean left foot acceler. in Z axis & 9 & $94.59 \%$ & $1.51 \%$ \\
\hline
\end{tabular}

TABLE IV

RESULTS OF NI DATASET CLASSIFICATION.

\begin{tabular}{|l|c|c|c|}
\hline \multicolumn{1}{|c|}{ Used parameter } & Neurons & Acc & Std \\
\hline all parameters & 2 & $100.00 \%$ & $0.00 \%$ \\
\hline $\begin{array}{l}\text { peak body dorsiflexion in stance phase, } \\
\text { knee range flexion, } \\
\text { peak body dorsiflexion in swing phase }\end{array}$ & 2 & $99.92 \%$ & $0.26 \%$ \\
\hline peak body dorsiflexion in stance phase & 9 & $81.91 \%$ & $3.25 \%$ \\
\hline peak body dorsiflexion in swing phase & 4 & $88.71 \%$ & $0.72 \%$ \\
\hline knee range flexion & 3 & $91.40 \%$ & $1.16 \%$ \\
\hline
\end{tabular}

$A c c=100 \%$ for the model with 2 hidden neurons. Having run the classifications process with a single parameter (mean right foot acceleration in $\mathrm{Z}$ axis) for single layer MLP, $100 \%$ accuracy is also obtained. Interestingly, $A c c=91.14 \%$ when using only mean acceleration right thigh in $\mathrm{Y}$ axis parameter with 3 neurons in hidden layer. Utilizing only the mean acceleration left foot in $\mathrm{Z}$ axis parameter, $94.59 \%$ accuracy is achieved for MLP with 9 hidden neurons.

\section{B. Result for NI dataset}

The classification with all 15 features allows MLP with 2 hidden neurons to achieve $100 \%$ accuracy. When using 3 most important features selected by the SA procedure, i.e.: peak body dorsiflexion in stance phase (100 importance), knee range flexion (61.90 importance), peak body dorsiflexion in swing phase (48.87 importance), Acc $=99.92 \%$ for MLP with 2 neurons in hidden layer. By employing only one parameter (peak body dorsiflexion in stance phase), 9 hidden neurons' MLP yields $81.91 \%$ of accuracy. Application of the knee flexion parameter allows MLP with 3 hidden neurons to reach $A c c=91.40 \%$. Consequently, the use of 4 neurons in hidden layer of the network with a single input parameter (peak body dorsiflexion in swing phase) provides the accuracy of $88.71 \%$.

\section{CONCLUSION}

In this article, the gait classification problem was studied. Three types of gait were registered: normal walk, tiptoeing and walk retaining long stance phase. For the purpose of the comparison, two data sets were created from the gait recordings. The first data set represented the statistical values calculated from 17 body segments. The second data set was composed of the NI parameters, which are commonly used in gait analyses. Furthermore, the SA procedure was applied to select the most important features in the data sets. The classification tasks were performed by MLP for both original, and reduced data. In all cases, the prediction accuracy achieved very high values at relatively simple architecture of MLP. Moreover, in some cases, decreasing the number of neurons in the hidden layer contributed to the increase of the accuracy.

The results presented in this work encourage the author to explore gait classification problem deeper. For this purpose, real gait recordings will registered from the patients in near future.

\section{REFERENCES}

[1] Statistics on parkinson's. Accessed: 2016-05-01. [Online]. Available: http://www.pdf.org/en/parkinson_statistics

[2] A. Ferrari, A. G. Cutti, P. Garofalo, M. Raggi, M. Heijboer, A. Cappello, and A. Davalli, "First in vivo assessment of "outwalk": a novel protocol for clinical gait analysis based on inertial and magnetic sensors," Medical \& biological engineering \& computing, vol. 48 , no. 1, pp. $1-15,2010$.

[3] A. Ferrari, "Technical innovations for the diagnosis and the rehabilitation of motor and perceptive impairments of the child with cerebral palsy," 2010.

[4] A. Szopa, M. Domagalska-Szopa, Z. Kidoń, and M. Syczewska, "Quadriceps femoris spasticity in children with cerebral palsy: measurement with the pendulum test and relationship with gait abnormalities," Journal of neuroengineering and rehabilitation, vol. 11, no. 1, p. 1, 2014.

[5] A. Martinez-Ramirez, P. Lecumberri, M. Gomez, and M. Izquierdo, "Wavelet analysis based on time-frequency information discriminate chronic ankle instability," Clinical Biomechanics, vol. 25, no. 3, pp. 256-264, 2010.

[6] H.-P. Brückner, W. Theimer, and H. Blume, "Real-time low latency movement sonification in stroke rehabilitation based on a mobile platform," in Consumer electronics (ICCE), 2014 IEEE international conference on, 2014, pp. 242-243.

[7] D. Morris, T. S. Saponas, A. Guillory, and I. Kelner, "Recofit: using a wearable sensor to find, recognize, and count repetitive exercises," in Proceedings of the SIGCHI Conference on Human Factors in Computing Systems. ACM, 2014, pp. 3225-3234.

[8] A. W. Lam, A. HajYasien, and D. Kulic, "Improving rehabilitation exercise performance through visual guidance," in Engineering in Medicine and Biology Society (EMBC), 2014 36th Annual International Conference of the IEEE. IEEE, 2014, pp. 1735-1738.

[9] Mvn biomech. Accessed: 2016-05-01. [Online]. Available: http: //www.xsens.com/products/mvn-biomech

[10] Matlab. Accessed: 2016-05-01. [Online]. Available: http://www. mathworks.com/products/matlab/

[11] Dtreg predictive modeling software. Accessed: 2016-05-01. [Online]. Available: http://www.dtreg.com

[12] Mvn studio software. Accessed: 2016-05-01. [Online]. Available: http://www.xsens.com/mvn-studio-pro

[13] M. Romei, M. Galli, F. Motta, M. Schwartz, and M. Crivellini, "Use of the normalcy index for the evaluation of gait pathology," Gait \& posture, vol. 19, no. 1, pp. 85-90, 2004.

[14] L. Schutte, U. Narayanan, J. Stout, P. Selber, J. Gage, and M. Schwartz, "An index for quantifying deviations from normal gait," Gait \& posture, vol. 11, no. 1, pp. 25-31, 2000.

[15] R. Tadeusiewicz, Sieci neuronowe. Akademicka Oficyna Wydawnicza Warszawa, 1993.

[16] J. Korbicz, A. Obuchowicz, and D. Uciński, "Artificial neural networks," AOW PLJ, Warsaw, 1994.

[17] D. E. Rumelhart, J. L. McClelland, and C. PDP Research Group, Eds., Parallel Distributed Processing: Explorations in the Microstructure of Cognition, Vol. 1: Foundations. Cambridge, MA, USA: MIT Press, 1986. 